\title{
Distinction between Epidermal Antigens Binding Pemphigus Vulgaris and Pemphigus Foliaceus Autoantibodies
}

John R. Stanley, Leena Koulu, and Charles Thivolet

Department of Dermatology, Uniformed Services University of the Health Sciences, Bethesda, Maryland 20814

bstract. Pemphigus vulgaris (PV) and pemphigus foliaceus (PF) are autoimmune blistering diseases in which antibodies develop to the cell surface of epidermal cells. In this study we sought to determine the antigenic specificity of antibodies in the sera of patients with PV and PF. Sera from 12 patients with PV were used to immunoprecipitate extracts of cultured human epidermal cells that were radiolabeled with ${ }^{14} \mathrm{C}$-amino acids. Immunoprecipitates were identified by SDS polyacrylamide gel electrophoresis (PAGE) and fluorography. All 12 PV sera precipitated a protein which, when reduced, displayed chains of 130,000 and 80,000 mol wt on SDSPAGE. Electrophoresis under nonreducing conditions identified a 210,000 -mol wt molecule, which was presumably formed by disulfide crosslinking of the 130,000 and $80,000-\mathrm{mol}$ wt chains. Immunoprecipitates of epidermal cell extracts that were labeled with ${ }^{14} \mathrm{C}$-glucosamine indicated that the 130,000 -mol wt chain was highly glycosylated compared with the 80,000-mol wt chain. Seven of eight PF sera, which were run concurrently with the PV sera in this immunoprecipitation assay, did not precipitate this glycoprotein, nor did they specifically precipitate any protein. To determine if a specific molecule which reacted with antibodies in PF sera could be identified, we used immunoblot analysis of extracts of normal human epidermis. The proteins in these extracts were reduced, separated by SDS-PAGE, and electrophoretically transferred to nitrocellulose sheets or to 2-aminophenylthioether paper. Immunoperoxidase staining of the transferred proteins with PF sera indicated that four of eight PF sera contained antibodies that stained a protein

Dr. Thivolet is the recipient of a grant from the Association of Cancer Research Development, Villejuif, France. Address all correspondence to Dr. Stanley.

Received for publication 3 November 1983 and in revised form 27 March 1984.

The Journal of Clinical Investigation, Inc.

Volume 74, August 1984, 313-320 band of $160,000 \mathrm{~mol} \mathrm{wt}$. Indirect immunofluorescence, using normal human skin as the substrate, indicated that IgG that was eluted from this protein band stained the epidermis in a cell surface pattern. PV sera did not specifically recognize any bands by immunoblot analysis. Immunoblots performed with PV antigen that was immunoprecipitated from cell culture extracts suggested that, once denatured for SDS-PAGE, PV antigen is no longer immunoreactive. Taken together, these data indicate that: autoantibodies contained in PV sera from various patients have a unique molecular specificity; autoantibodies from most PF sera have a specificity different from that of PV autoantibodies; and autoantibodies from various PF patients may not have identical antigenic specificities. These differences in antigenic specificity between PV and PF sera may account for the clinical and histologic differences between these diseases.

\section{Introduction}

Pemphigus is an autoimmune blistering disease of the skin. The histopathology of this disease demonstrates that blisters occur because of a loss of cell-to-cell adhesion, a process called acantholysis, which is presumably caused by autoantibodies (1-7). There are at least two types of pemphigus, as defined by clinical and histologic criteria $(1,2)$. Pemphigus vulgaris (PV), the most common type of pemphigus, is characterized by flaccid blisters and erosions in a generalized distribution, including the mucous membranes, which are almost always involved. Histopathology of PV lesions indicates acantholysis with the resultant blister formation deep in the epidermis, just above the basal layer. Pemphigus foliaceus (PF) is characterized by crusted and scaly lesions, which are commonly distributed on the scalp, face, upper chest, and back. Mucous membrane involvement is unusual. Histopathology of PF lesions demonstrates acantholysis and blister formation in the superficial epidermis, at the level of the granular layer.

1. Abbreviations used in this paper: APT, 2-aminophenylthioether; PF, pemphigus foliaceus; PV, pemphigus vulgaris; PAGE, polyacrylamide gel electrophoresis; TBS, $0.15 \mathrm{M} \mathrm{NaCl}, 10 \mathrm{mM}$ Tris- $\mathrm{HCl} \mathrm{pH} 7.4$. 
In both PV and PF, biopsies from patients, when examined by direct immunofluorescence, demonstrate IgG on the cell surface of epidermal cells (1-3). In addition, most patients with pemphigus have circulating antibodies (IgG) which bind the surface of cells in normal epidermis (or other stratified squamous epithelia), as detected by indirect immunofluorescence (1-3). Although a few patients with PF have been reported to have antibodies that bind only in the superficial epidermis, in general, antibodies from patients with PV and PF bind the cell surface of cells throughout the epidermis in patterns that are indistinguishable $(8,9)$.

These autoantibodies are pathogenic in both PV and PF. PV IgG transferred to newborn mice causes the pathologic lesions of pemphigus (4). In vitro models using organ culture of skin have demonstrated that PV or PF IgG, in the absence of complement or inflammatory cells, can cause acantholysis (5-7). It is thought that the pemphigus IgG bound to the surface of the epidermal cell causes the release of one or more proteolytic enzymes, which results in acantholysis (7, 10-14).

Although the importance of antibody binding to the epidermal cell surface in triggering the pathology of pemphigus has been well established, it is not known whether antibody binding to specific cell surface antigens is required to induce the specific pathology of PV or PF. Our recent studies demonstrated that most, but not all, pemphigus sera immunoprecipitate a glycoprotein synthesized by epidermal cells in culture (15). This molecule, when radiolabeled with ${ }^{14} \mathrm{C}$-glucosamine and reduced, has a chain of $130,000 \mathrm{~mol} \mathrm{wt}$, as identified by SDS polyacrylamide gel electrophoresis (PAGE). The finding that not all pemphigus sera react with this molecule suggests that there may be heterogeneity in the cell surface molecules reactive with pemphigus antibodies. It is not known if either PV or PF antibodies react with this glycoprotein and, in general, if $\mathrm{PV}$ and $\mathrm{PF}$ antibodies have distinct and unique antigenic specificities.

In the present study we show that it is the PV sera that bind the glycoprotein we have previously identified, demonstrate that PV sera from different patients all bind this glycoprotein, and further characterize the glycoprotein as a molecule with $\sim 210,000$ mol wt which, when reduced, has chains of 130,000 and $80,000 \mathrm{~mol}$ wt. In addition, we demonstrate that most PF sera are distinct from PV sera in their antigenic specificity. Some, but not all, PF sera react with a protein which, when reduced, has $\sim 160,000$ mol wt.

\section{Methods}

Sera. Sera from 12 patients with PV, 8 patients with PF, and 11 normals were used. In 11 of 12 patients with PV and all patients with PF, the diagnosis was confirmed histologically. PV biopsies demonstrated suprabasilar clefts with acantholysis, and PF biopsies showed subcorneal clefts with acantholysis, with or without neutrophilic infiltration. The diagnosis of one patient with PV was based on typical clinical findings (including extensive oral erosions) and positive indirect immunofluorescence. All pemphigus sera, when tested by indirect immunofluores- cence (16), contained IgG which bound, in a cell surface pattern, throughout the epidermis of normal human skin or the epithelium of monkey esophagus, at titers of $>40$. The indirect immunofluorescence titers of the PV and PF sera were equivalent. The patterns of cell surface staining of the PV and PF sera were indistinguishable.

Metabolic radiolabeling and extraction of epidermal cell cultures. Human epidermal cells, derived from neonatal foreskins, were cultured either on collagen substrates, as previously described $(15,17)$, or on 3T3 fibroblast feeder layers (18-20). Cells were used in primary or first passage culture and, at the time of radiolabeling, were just confluent with slight stratification visible in some areas. In order to radiolabel pemphigus antigen for immunoprecipitation experiments, a mixture of ${ }^{14} \mathrm{C}$-amino acids (New England Nuclear, Boston, MA) at $12.5 \mu \mathrm{Ci} / \mathrm{ml}$ was added to the cells for 18-24 h. For cells grown on collagen substrates, labeling was done in basal medium Eagle (M.A. Bioproducts, Walkersville, MD), and for cells grown on 3T3 feeder layers, radiolabel was added to the same medium that was used to grow the cells-Dulbecco's modified Eagle medium (Gibco, Grand Island, NY) supplemented with $0.4 \mu \mathrm{g} / \mathrm{ml}$ hydrocortisone (Sigma Chemical Co., St. Louis, MO), $10^{-10}$ M cholera toxin, $15 \mu \mathrm{g} / \mathrm{ml}$ epidermal growth factor, $5 \mu \mathrm{g} / \mathrm{ml}$ bovine insulin, $5 \mu \mathrm{g} / \mathrm{ml}$ human transferrin, all from Sigma Chemical Co., and $10 \%$ fetal bovine serum $(19,20)$. The cell layer was extracted, after radiolabeling, with $0.5 \%$ Nonidet $\mathrm{P}-40$, as previously described (15). This radiolabeled extract was used for immunoprecipitation experiments.

Immunoprecipitation. To identify and characterize newly synthesized pemphigus antigen in cultured cells, we used a protein A-immunoprecipitation technique that was previously described $(15,21)$. In brief, after preabsorption with normal human serum and protein A-bearing staphylococci (Calbiochem-Behring Corp., San Diego, CA), an aliquot of the cell extract was incubated with $5 \mu$ l of either pemphigus or normal sera (different from the one used in the preabsorption) at $4^{\circ} \mathrm{C}$ overnight. Protein A-bearing staphylococci were added to precipitate the antigen-antibody complexes. The pellet was washed six times with $0.3 \%$ Nonidet $\mathrm{P}-40,0.3 \%$ sodium deoxycholate, $0.3 \mathrm{M} \mathrm{NaCl}$ in $10 \mathrm{mM}$

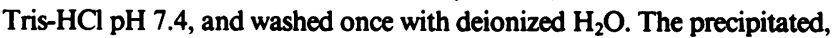
washed, antigen-antibody complexes were then solubilized by incubating at $100^{\circ} \mathrm{C}$ with gel sample buffer ( $2 \%$ SDS, $0.0625 \mathrm{M}$ Tris- $\mathrm{HCl}, \mathrm{pH} 6.8$ ) with or without $0.1 \mathrm{M}$ dithiothreitol for reduced and unreduced samples, respectively. Specifically precipitated antigens were then identified on SDS-PAGE $(22)$ by fluorography $(23,24)$ using Enhance (New England Nuclear). Approximate molecular weights of immunoprecipitated molecules were determined on 5, 6, and $7.5 \%$ acrylamide gels by using either Coomassie Blue stained high molecular standards (Bio-Rad Laboratories, Richmond, CA) or ${ }^{14} \mathrm{C}$-labeled standards (New England Nuclear). Specific standards used were myosin, $\beta$-galactosidase, phosphorylase $B$, and bovine serum albumin.

Extraction of normal human epidermis and immunoblotting. To identify antigens that are present in normal human skin, we extracted suction blister-derived epidermis (from volar forearm) with gel sample buffer, as previously described (25). The extracted proteins were separated by SDS-PAGE, then electrophoretically transferred to either nitrocellulose, as previously described $(25,26)$, or to 2-aminophenylthioether (APT) paper (Bio-Rad Laboratories), which binds proteins covalently. Electrophoretic transfer to APT paper was performed according to the supplier's instructions. In short, paper was activated (diazotized) by incubation for $30 \mathrm{~min}$ at $4^{\circ} \mathrm{C}$ in a solution of $0.03 \%$ sodium nitrite in 1.2 M HCl. Electrophoretic transfer from gel to paper was then performed in $25 \mathrm{mM}$ sodium phosphate buffer, $\mathrm{pH} 6.5$, using a Trans-Blot cell (Bio-Rad Laboratories) at $50 \mathrm{~V}$ for 4-6 h. A cooling coil with recirculating water at $4^{\circ} \mathrm{C}$ was used for cooling during the transfer. After transfer, 
free protein binding sites were blocked by incubating the paper in $1 \%$ glycine in $0.15 \mathrm{M} \mathrm{NaCl}, 10 \mathrm{mM}$ Tris- $\mathrm{HCl}, \mathrm{pH} 7.4$ (TBS) overnight at $4^{\circ} \mathrm{C}$. Antigens on either nitrocellulose or APT paper were identified by immunoperoxidase staining, as previously described (25). Pemphigus sera (and normal human sera controls) were used in dilutions of 1:1001:250.

Elution of antibodies from immunoblots. To confirm that PF antibodies, which specifically bound to a 160,000 -mol wt protein band on immunoblots, were actually pemphigus antibodies, we eluted the IgG from the protein that was covalently bound to the APT paper, and used the eluted antibodies for indirect immunofluorescence on normal human skin to demonstrate cell surface staining of the epidermis. A technique similar to that described by Olmsted (27) was used. 15 lanes of electrophoresed proteins extracted from suction blister epidermis were transferred to one sheet of APT paper. The first and last lanes were cut off (as vertical strips) and immunoperoxidase staining was performed to identify the PF antigen in these lanes. These two stained lanes were then used to locate the unstained PF antigen in the 13 lanes left on the APT paper. These unstained protein bands were cut out in a horizontal strip. Thus, the strip contained 13 bands of PF antigen. A control strip of the same width (from below the area where the PF antigen was located) was also cut out. Each of these two horizontal strips was then incubated at $4^{\circ} \mathrm{C}$ overnight with a PF serum (175) that was diluted 1:50 (total volume $2.5 \mathrm{ml} / \mathrm{strip}$ ). The strips were washed two times with washing buffer $(10 \mathrm{mM}$ Tris- $\mathrm{HCl}, 0.25 \mathrm{M} \mathrm{NaCl}, 0.5 \%$ Nonidet P-40, $\mathrm{pH}$ 7.4) and three times with TBS. The bound antibodies were then eluted twice with $0.5 \mathrm{M}$ acetic acid, $0.5 \mathrm{M} \mathrm{NaCl}, \mathrm{pH} 2.5$ (total volume $5.0 \mathrm{ml} / \mathrm{strip}$ ). Each elution was for $15 \mathrm{~min}$ at room temperature. The eluted antibodies were neutralized immediately using $2 \mathrm{M}$ Tris- $\mathrm{HCl}$, $\mathrm{pH} 8.5,1.1 \mathrm{ml}$ for each $2.5 \mathrm{ml}$ of eluant. After elution the horizontal strips were washed with $3 \%$ bovine serum albumin in washing buffer and with 10\% normal goat serum in TBS, and the antibody binding and elution were repeated. The eluted, neutralized antibodies were dialyzed against phosphate-buffered saline and concentrated on an Amicon PM-10 filter (Amicon Corp., Lexington, MA) to $\sim 1.5 \mathrm{ml}$. Bovine serum albumin and sodium azide were added to a concentration of 0.1 and $0.02 \%$, respectively, and the antibodies were further concentrated on a Minicon B15 unit (Amicon Corp.) to $\sim 100 \mu$ l. These antibodies were then used for indirect immunofluorescence.

\section{Results}

Different $P V$ sera immunoprecipitate the same glycoprotein from extracts of human epidermal cell culture. In order to identify PV antigen that was extracted from cultured human epidermal cells, we used sera from 12 patients with PV in immunoprecipitation assays. Proteins synthesized by human epidermal cells in culture were radiolabeled with ${ }^{14} \mathrm{C}$-amino acids, and immunoprecipitated proteins were identified by SDS-PAGE and fluorography (to visualize radiolabeled proteins). All 12 PV sera precipitated a protein which, when reduced, had chains of $\sim 130,000$ and 80,000 mol wt (Fig. 1 shows seven of these PV sera). The 80,000 -mol wt band often appeared as a doublet with the slightly higher molecular weight band being the more intense. The specificity of this immunoprecipitation by PV sera is demonstrated by the finding that none of the 11 normal human sera, used concurrently with the PV sera in various immunoprecipitation experiments, precipitated these chains (Fig. 1 in-

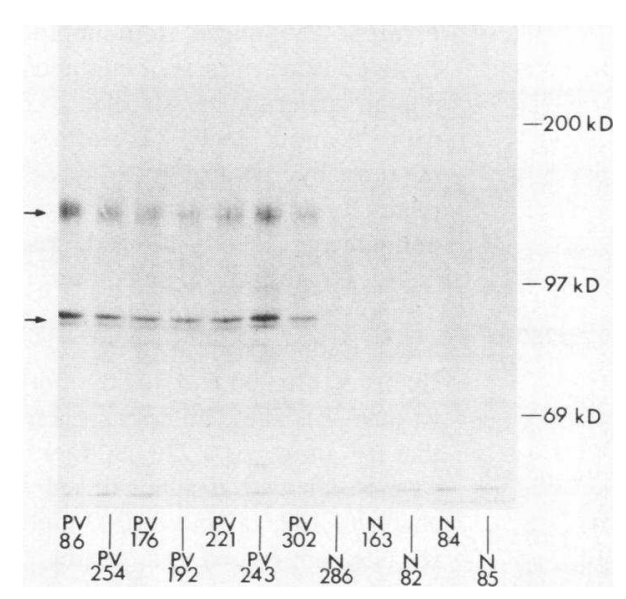

Figure 1. Antigenic specificity of PV sera from different patients. PV antigen was immunoprecipitated from extracts of cultured human epidermal cells that were radiolabeled with ${ }^{14} \mathrm{C}$-amino acids. Immunoprecipitates were reduced and electrophoresed by SDS-PAGE. Radiolabeled antigens were then identified by fluorography. Each lane shows radiolabeled proteins that were precipitated with PV serum or normal serum (N). Different numbers indicate sera from different patients or normals. All PV sera specifically precipitated protein chains of $\sim 130,000$ and 80,000 mol wt (arrows). The 80,000 -mol wt chain was usually seen as a doublet, with the higher band more intense, as demonstrated here. Normal sera did not precipitate these chains. Other bands that were seen on this fluorograph were precipitated by both PV serum and $\mathrm{N}$ serum, and therefore are considered to be nonspecific. The migration of molecular weight markers is indicated on the right. $\mathrm{kD}$, molecular weight $\times 10^{-3}$.

cludes five of these normal sera). In addition, five bullous pemphigoid sera, used concurrently with the PV sera in some experiments, did not precipitate these chains. If the immunoprecipitates were electrophoresed unreduced, a protein of $\sim 210,000$ mol wt was demonstrated by SDS-PAGE (Fig. 2). This $210,000-\mathrm{mol}$ wt molecule was precipitated by all of the four different PV sera tested under nonreducing conditions. These findings suggest that PV antigen consists of two chains of 130,000 and $80,000 \mathrm{~mol} \mathrm{wt}$, which are disulfide bonded in a $210,000-\mathrm{mol}$ wt molecule.

The co-migration, under reducing conditions, of the 130,000 mol wt bands and the 80,000-mol wt doublets, and, under nonreducing conditions, of the $210,000-\mathrm{mol}$ wt bands that were precipitated by different PV sera, strongly suggest that autoantibodies in PV sera from different patients bind the same molecule. Additional evidence for the common molecular specificity of different PV sera was obtained by performing sequential immunoprecipitation with different PV sera (Fig. 3).

We have previously demonstrated that, if ${ }^{14} \mathrm{C}$-glucosamine is used to label human epidermal cell cultures, most pemphigus sera precipitate a molecule with a 130,000 -mol wt chain, under reducing conditions (15). We also demonstrated that this chain was a glycoprotein, and that different pemphigus sera which reacted with this chain were directed against the same molecule. 


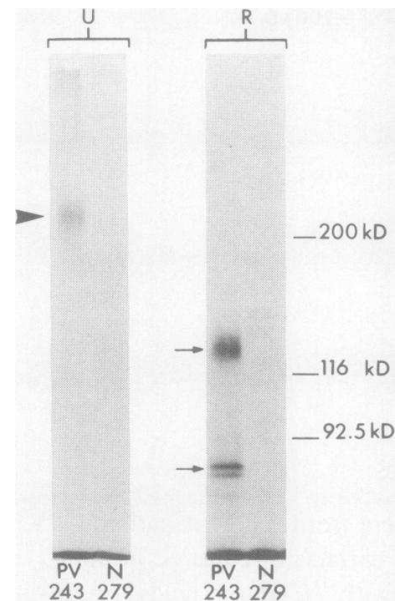

Figure 2. PV antigen, immunoprecipitated from extracts of cultured human epidermal cells labeled with ${ }^{14} \mathrm{C}$-amino acids, was electrophoresed by SDS-PAGE (5\% acrylamide) under reducing (R) and nonreducing $(U)$ conditions. Unreduced, $\mathrm{PV}$ antigen migrated as a 210,000-mol wt protein (arrowhead). Reduction resulted in chains of 130,000 and $80,000 \mathrm{~mol}$ wt (arrows). These findings suggest that PV antigen is a $210,000-\mathrm{mol}$ wt molecule with disulfide-linked chains of 130,000 and $80,000 \mathrm{~mol}$ wt. $\mathbf{N}$ serum is used as a control to demonstrate that the PV chains indicated are specifically precipitated. $\mathrm{kD}$, molecular weight $\times 10^{-3}$.

To show that the $130,000-\mathrm{mol}$ wt chain seen in the present experiments, which used ${ }^{14} \mathrm{C}$-amino acids as the label, was the same as the 130,000 -mol wt chain in experiments using ${ }^{14} \mathrm{C}$ glucosamine as the label, we radiolabeled identical cultures with either ${ }^{14} \mathrm{C}$-amino acids or ${ }^{14} \mathrm{C}$-glucosamine. Starting with equal amounts of radioactivity, we immunoprecipitated both extracts with the same PV sera. Fig. 4 shows that the $130,000-\mathrm{mol}$ wt chain is specifically precipitated using both extracts, but that the 80,000 -mol wt band does not label well with ${ }^{14} \mathrm{C}$-glucosamine. This finding suggests that the 130,000 -mol wt chain is more heavily glycosylated than the 80,000 -mol wt chain. When the immunoprecipitates were electrophoresed unreduced (not shown), the 210,000-mol wt glycoprotein was labeled with both ${ }^{14} \mathrm{C}$-amino acids and ${ }^{14} \mathrm{C}$-glucosamine, but it was labeled less well with ${ }^{14} \mathrm{C}$-glucosamine. This finding is consistent with the

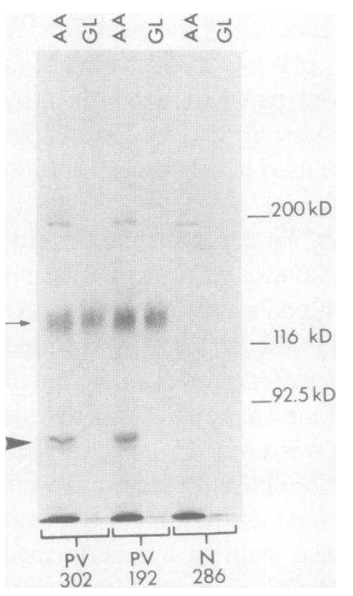

Figure 4. $\mathrm{PV}$ antigen radiolabeled with ${ }^{14} \mathrm{C}$-glucosamine $(\mathrm{GL})$ and ${ }^{14} \mathrm{C}$ amino acids (AA). Immunoprecipitations of extracts of cultured human epidermal cells that were radiolabeled with either ${ }^{14} \mathrm{C}$-glucosamine or ${ }^{14} \mathrm{C}$ amino acids were performed, starting with equal amounts of radioactivity. The 130,000-mol wt band (arrow) of PV antigen labeled well with both, whereas the 80,000-mol wt band (arrowhead) did not label with ${ }^{14} \mathrm{C}$-glucosamine. The findings demonstrate that the 130,000 -mol wt chain is heavily glycosylated compared with the 80,000-mol wt chain. Normal (N) serum is used as a control to demonstrate that the PV chains which are indicated are specifically precipitated. $\mathrm{kD}$, molecular weight $\times 10^{-3}$.

assumption that the $210,000-\mathrm{mol}$ wt molecule consists of the highly glycosylated $130,000-\mathrm{mol}$ wt protein and the poorly glycosylated 80,000-mol wt protein.

Most $P V$ and $P F$ sera demonstrate different major antigenic specificities. We next wanted to determine whether antibodies from PF sera had the same antigenic specificity as the PV sera. Sera from eight patients with PF were tested, concurrent with PV sera, in the immunoprecipitation assay described above. Whereas all $12 \mathrm{PV}$ sera that were tested consistently precipitated the 130,000-mol wt and 80,000-mol wt chains from extracts of ${ }^{14} \mathrm{C}$-amino acid-labeled human epidermal cell cultures, only one of the eight PF sera precipitated these chains. (Fig. 5 shows a typical experiment in which two PV sera precipitate these chains whereas two PF sera do not.) Sequential immunoprecipitation

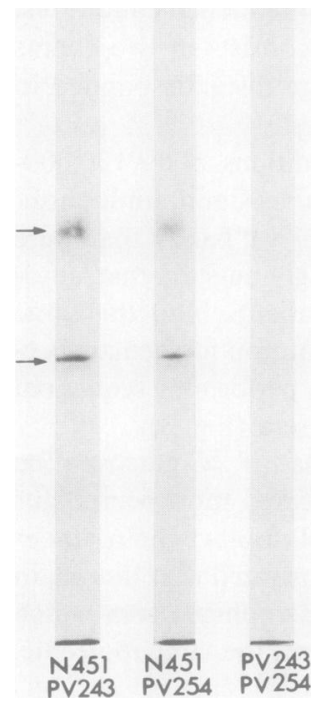

Figure 3. Sequential immunoprecipitation which demonstrates that different PV sera bind the same molecule. Under each lane is listed first the serum used in a preliminary immunoprecipitation (gel not shown) of an extract of cultured human epidermal cells that were radiolabeled with ${ }^{14} \mathrm{C}$-amino acids. Listed second under each lane is the serum that was used in an immunoprecipitation of the supernatant of the preliminary incubation. This second immunoprecipitate was electrophoresed, and the fluorograph of the gel is shown. The results indicate that reaction of the cell extract with one PV serum (as opposed to normal (N) serum) markedly depleted the amount of antigen reactive with a different $\mathrm{PV}$ serum, which confirmed that different PV sera bind the same molecule. Arrows indicate bands of PV antigen.

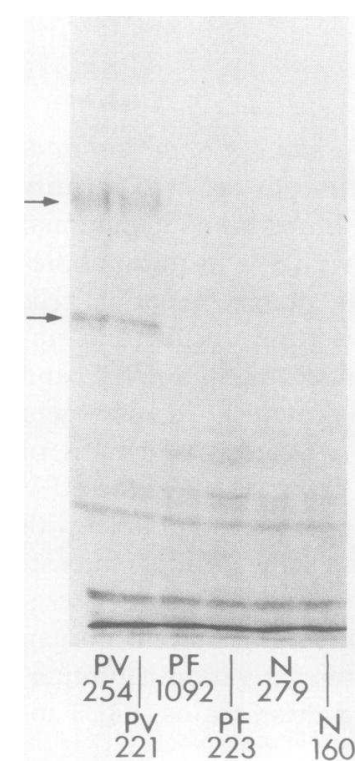

Figure 5. Most PF sera do not immunoprecipitate the PV antigen. Immunoprecipitates of extracts of cultured human epidermal cells that were labeled with ${ }^{14} \mathrm{C}$-amino acids were performed, starting with equal amounts of radioactivity. The PV sera, but not the PF sera (or normal [N] sera), precipitated the chains of PV antigen (arrows). These results are not due to differences in antibody titer of the sera used. For example, when PV 254, PV 221, and PV 1092 were titered by indirect immunofluorescence on human neonatal foreskin (the source of cells used in these experiments), the titers were 80,640 , and 320 , respectively. Of the eight PF sera used in various experiments, only one (Fig. 6) precipitated the chains of PV antigen. 
confirmed that the one exceptional PF serum (275) actually precipitated the PV antigen (Fig. 6). The other seven PF sera did not precipitate the PV antigen or any other detectable specific molecules, even if the human epidermal cells are induced to more fully differentiate by depletion of the vitamin A from the fetal bovine serum that was used in the culture medium $(19,20)$.

These results demonstrate that whereas PV sera from different patients share antigenic specificity and define a specific glycoprotein that is synthesized by epidermal cells in culture, the PF sera tested, with one exception, do not share this particular antigenic specificity.

We next determined whether we could identify a molecule to which antibodies from PF sera would bind. Because we were unable to detect PF antigen in radiolabeled extracts of cultured human epidermal cells, we used extracts of normal human epidermis, which were derived from suction blisters, in an immunoblot assay to identify proteins that were reactive with PF autoantibodies. This procedure does not depend on synthesis and metabolic labeling in vitro. The extracted proteins were separated by SDS-PAGE (under reducing conditions), and were electrophoretically transferred to either nitrocellulose sheets or APT paper, which binds proteins covalently. The lanes of protein were then cut out and used for immunoperoxidase staining with PF sera. Four of eight PF sera, including the one that precipitated the PV antigen from cultured cells, specifically stained a 160,000 mol wt protein (Fig. 7 shows three of these sera). All eight normal human sera that were tested, when used concurrently in various experiments, did not stain this band. In general, PV sera, tested concurrently, did not stain this 160,000 -mol wt band (Fig. 7), nor did they specifically stain any other band (see below). However, three of these nine PV sera inconsistently demonstrated an extremely faint staining of a band in the area of $160,000 \mathrm{~mol} \mathrm{wt}$ if these sera were used at high concentrations

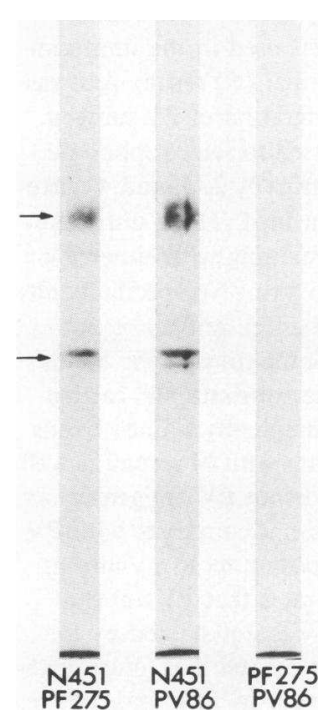

Figure 6. Sequential immunoprecipitation demonstrates that one exceptional PF serum (275) binds the same molecule as PV serum. (See legend Fig. 3 for experimental details.) The results indicate that reaction with PF 275 (as opposed to normal [N] serum) markedly depleted the amount of antigen reactive with PV 86. Thus, PF 275 precipitates the same molecule as PV serum. Seven other PF sera that were tested in other experiments did not immunoprecipitate the PV antigen. Arrows indicate PV antigen.

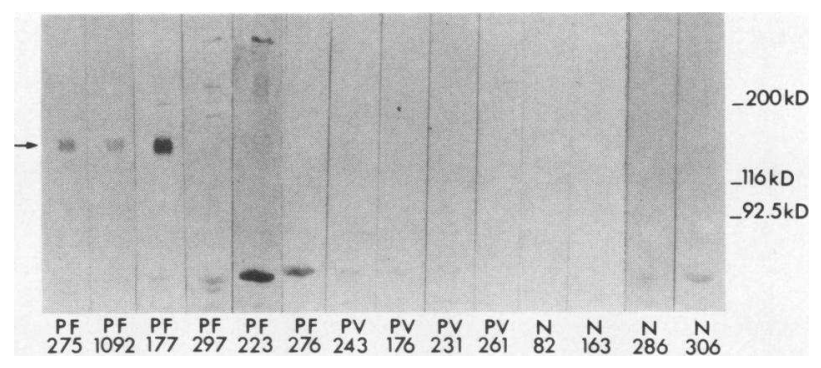

Figure 7. Immunoblot analysis demonstrates that antibodies from certain PF sera bind a 160,000 -mol wt protein that was extracted from normal human epidermis. Proteins in extracts of normal human epidermis were reduced, separately by SDS-PAGE, and electrophoretically transferred to APT paper, which binds protein covalently. Individual lanes of protein on APT paper were stained using an immunoperoxidase technique with PF, PV, or normal (N) sera. Three of the PF sera shown here specifically stained a band at 160,000 mol wt. The other PF sera, the PV sera (and the $\mathrm{N}$ sera) did not stain this band. These results are not due to differences in antibody titer of the sera used. The indirect immunofluorescence titers (using monkey esophagus or adult normal human skin as substrate) of the sera used were $1,280,640,160,320,>320,40,320,160$, $>640,160$ for PF 275, PF 1092, PF 177, PF 297, PF 223, PF 276, PV 243, PV 176, PV 231, and PV 261, respectively. Other bands that were seen in this experiment were either nonspecifically stained (as shown by the staining with $\mathrm{N}$ sera) or were not consistently stained in additional experiments. These studies suggest that PF sera from different patients may have different antigenic specificities. $k D$, molecular weight $\times 10^{-3}$.

or if development in peroxidase substrate was extensive. The staining of these three PV sera was erratic from experiment to experiment, and was clearly different from the specific and intense staining seen with the PF sera. We did not consider the staining by these three PV sera as significant; however, it is possible that this faint staining may indicate a minor crossreactivity of antibodies in some $P V$ sera with the antigen defined by the PF sera.

To confirm that the PF antibodies that bound to the 160,000 mol wt protein were, in fact, pemphigus antibodies, we eluted the antibodies from the 160,000 -mol wt protein that was covalently bound to APT paper. These eluted antibodies, when used for indirect immunofluorescence of normal human skin, displayed the typical cell surface staining of the epidermis that was seen with pemphigus antibodies (Fig. 8). No cell surface staining was detected with a control eluate from an area of the same APT paper below the 160,000 -mol wt band.

These results demonstrate that the autoantibodies in PF sera from different patients may have different antigenic specificities, but that at least some patients with PF have in common antibodies which bind a specific molecule that is extractable from normal human epidermis. These findings, taken together with the immunoprecipitation results, indicate that the major antigenic specificities of almost all PF and PV autoantibodies are distinct. The one exception was a PF serum that immunoprecipitated the PV antigen from extracts of cultured cells but that 


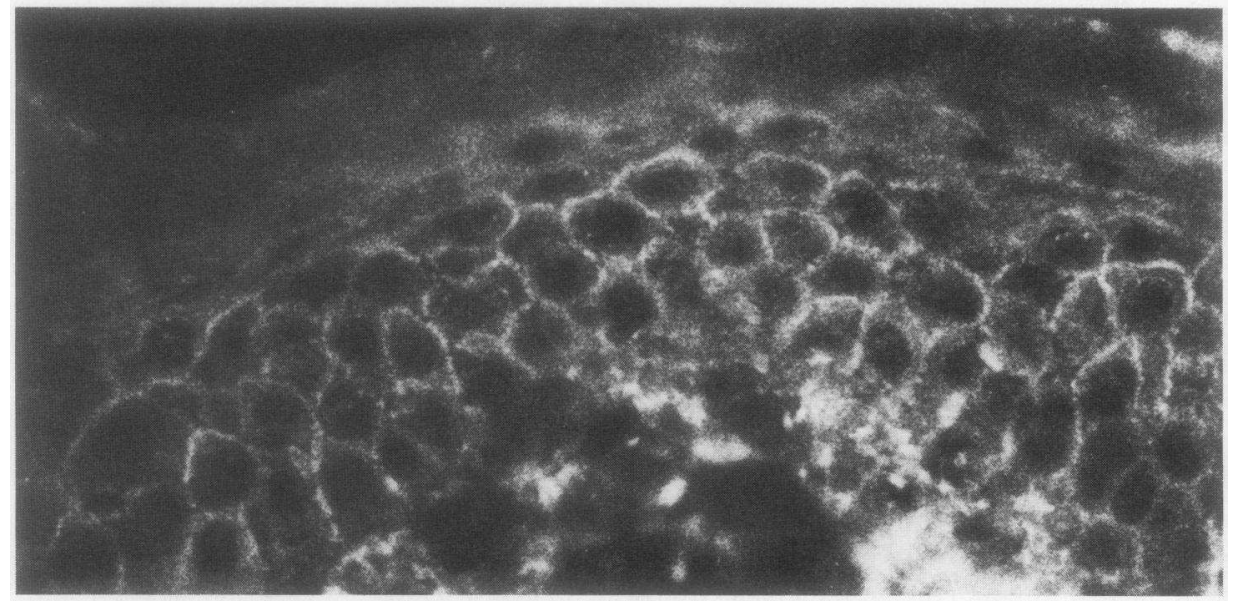

Figure 8. Indirect immunofluorescence on normal human skin demonstrates that PF antibodies which bind the 160,000 -mol wt protein band on immunoblots also bind the cell surface of epidermal cells. Extracts of normal human skin were electrophoresed by SDSPAGE, then transferred to APT paper. The paper was subsequently incubated with PF serum, and the antibodies that bound to the 160,000 -mol wt band were eluted. These antibodies were used for indirect immunofluorescence, and demonstrate the pattern of cell surface fluorescence that is normally seen with pemphigus sera. $(\times 460)$

also stained the $160,000-\mathrm{mol}$ wt molecule on immunoblots. Whether this serum contained antibodies of different antigenic specificities or whether these antibodies were capable of binding both antigens is not known.

$P V$ antigen cannot be identified by immunoblotting by using extracts of either normal human epidermis or human epidermal cell culture. To confirm that PV sera did not specifically stain any protein bands on immunoblots which used extracts of normal human epidermis, we attempted to detect PV antigen by immunoblotting using the following conditions: normal human epidermis was extracted with gel sample buffer or $0.5 \%$ Nonidet P-40 (which extracts PV antigen from cultured cells, as demonstrated by immunoprecipitation experiments); proteins were separated on 5, 7.5, and 5-12\% gradient gels before transfer; and proteins were transferred to nitrocellulose or APT paper. In none of these situations were we able to detect PV antigen by immunoperoxidase staining of the transferred proteins.

In an attempt to determine why we were unable to detect PV antigen by immunoblot analysis of extracts of human epidermis, we investigated whether PV antigen that was immunoprecipitated from cell culture could be identified by immunoblot analysis. PV antigen, radiolabeled with ${ }^{14} \mathrm{C}$-amino acids, was immunoprecipitated from extracts of cultured human epidermal cells. This immunoprecipitate was run by SDS-PAGE in duplicate. Fluorography of the gel (Fig. $9 \mathrm{~A}$ ) shows that PV antigen was present in the gel. The duplicate gel was then used for electrophoretic transfer of the PV antigen to nitrocellulose strips. Fluorography was performed on this gel after transfer, and the PV antigen was no longer detectable. This finding demonstrates that PV antigen can be transferred out of the gel. The transferred lanes of protein on nitrocellulose were used for immunoperoxidase staining with the same PV serum that was used to precipitate the antigen, and a normal human serum control was also used. No specific bands were stained (Fig. $9 \mathrm{~B}$ ). Then, in order to determine whether the PV antigen, which had transferred out of the gel, had actually bound to the nitrocellulose strips, we fluorographed the nitrocellulose strips. PV antigen was detected bound to the nitrocellulose (Fig. $9 \mathrm{C}$ ) even though it had not bound the PV antibodies, as indicated by the negative immunoperoxidase staining. These data suggest that once denatured (for running by SDS-PAGE), the PV antigen from culture is no longer immunoreactive. Given this data, and assuming

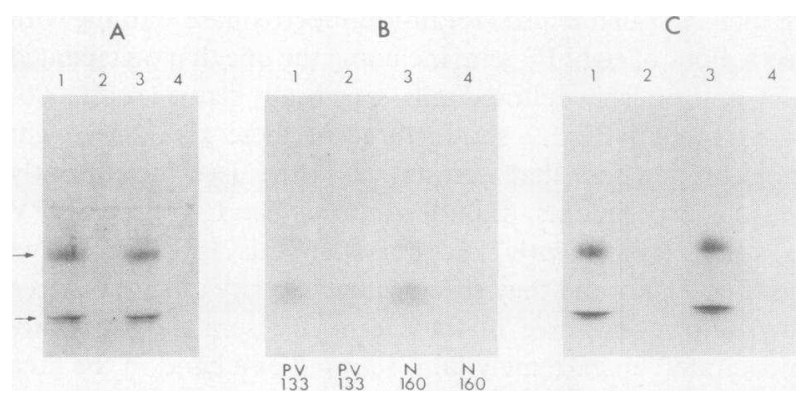

Figure 9. PV antigen, which has been transferred to nitrocellulose, cannot be detected by immunoperoxide staining. $(A)$ Immunoprecipitates of extracts of cultured human epidermal cells that were radiolabeled with ${ }^{14} \mathrm{C}$-amino acids were identified by SDS-PAGE and fluorography. In lanes 1 and 3, PV serum 133 was used in the immunoprecipitation assay, and in lanes 2 and 4, normal (N) serum 160 was used as a control. Arrows indicate relative migration of PV antigen. (B) A duplicate gel to that shown in $A$ was used for electrophoretic transfer of proteins to nitrocellulose strips. Strips 1, 2, 3, and 4 correspond to lanes 1, 2, 3, and 4 that were shown in $A$. These nitrocellulose strips were used for immunoperoxidase staining with either PV serum or $\mathrm{N}$ serum, as indicated beneath each strip. No specific bands were stained by PV serum, which indicates that PV antigen is not recognized by this immunoblot technique. (Some nonspecific bands are visible because development in peroxidase substrate was carried out extensively to be sure to visualize even marginally stained bands. These bands are nonspecific because: they stain with $\mathrm{N}$ serum as well as with PV serum, they are present whether or not PV antigen is bound to the nitrocellulose strip, and they do not comigrate with PV antigen.) $(C)$ Fluorography (same time of exposure to X-ray film as in $A$ ) of the same strips shown in $B$ demonstrates that PV antigen was present in lanes 1 and 3, even though it was not stained by the immunoperoxidase technique. These findings suggest that, once denatured for SDS-PAGE, PV antigen may no longer by immunoreactive. 
that the antigenic sites recognized by PV autoantibodies in vivo are similar to those in vitro, we speculate that the most likely reason for failure to detect $\mathrm{PV}$ antigen extracted from normal epidermis by immunoblot analysis is that PV antigen, once denatured, is no longer immunoreactive.

\section{Discussion}

In a previous study we showed that most, but not all, pemphigus sera bind an epidermal cell surface glycoprotein with a chain of $130,000 \mathrm{~mol} \mathrm{wt}(15)$. This glycoprotein was synthesized by cultured mouse and human keratinocytes. In the present study, we expand those findings to demonstrate that: this glycoprotein reacts with antibodies in all 12 PV sera tested but in only one of eight PF sera tested; and the glycoprotein consists of two chains: a highly glycosylated 130,000 -mol wt chain and a poorly glycosylated 80,000 -mol wt chain, which are probably disulfidebonded in a $210,000-\mathrm{mol}$ wt molecule. We also defined a molecule reactive with PF autoantibodies by using immunoblot analysis of extracts of normal human epidermis. Four of eight PF sera contained antibodies which bound a 160,000-mol wt molecule. Antibodies eluted from this band, when used in an immunofluorescence assay, stained the epidermis in a cell surface pattern, as do pemphigus antibodies. In contrast to the findings with PF sera, PV sera did not specifically stain any bands by immunoblot analysis. Taken together, these data demonstrate that: PV autoantibodies have a common antigenic specificity on a molecular level; most PF autoantibodies do not bind the PV antigen; and autoantibodies from different PF patients may have different antigenic specificities, but, at least, some share a common specificity. Although most PV and PF autoantibodies have different major antigenic specificities, there may be individual sera that bind to both PV and PF antigens (e.g., one PF serum immunoprecipitated the PV antigen and bound to the 160,000-mol wt molecule on immunoblot analysis). However, in our experience thus far, this crossreactivity is the exception.

The PV antigen which we have characterized is from human epidermal cell culture. Since it is unlikely that various patients with PV would develop autoantibodies to an antigen expressed only by cultured cells, the antigen defined by PV sera in vitro should be immunologically very similar to the antigen found in skin. However, the exact molecular nature of the antigen in skin and culture may be different if, for example, posttranslational modification in vivo is different than in vitro. Several previous studies have attempted to define the PV antigen that is extracted from tissue (28-30). These studies reported molecules of varying molecular weights, all lower than that of the antigen which we extracted from cultured cells. These disparate results may be due to several factors: ( $a$ ) the type of pemphigus serum used to characterize the antigen was not defined $(28) ;(b)$ different sources for extraction were used (e.g., saliva [28], guinea pig skin [29], human esophagus [30]); (c) possible proteolytic breakdown during extensive chromatography procedures $(28,29)$; and $(d)$ partial purification that resulted in a mixture of substances $(29,30)$. The exact molecular nature of the PV antigen in vivo remains to be determined.
The different antigenic specificities between PV and PF antibodies demonstrated here has also been suggested by previous studies. Immunofluorescence blocking studies performed with sera from four patients with PV and two patients with Brazilian PF (a form of PF endemic to certain areas of Brazil) suggested that PV and PF antibodies bind different antigenic determinants (9). In addition, the report that, by indirect immunofluorescence, some PF sera bound the epidermis in a different pattern than PV sera also, indicates that, at least for those PF sera, there may be an antigenic specificity different from that of PV sera (8). However, antigenic masking may also account for different immunofluorescent patterns of identical antigens. In addition, most PF sera display the same pattern as PV sera in indirect immunofluorescence assays. The present study demonstrates that even if the PV and PF sera stain the epidermis in an indistinguishable pattern, they can have distinct antigenic specificities. We think it likely that the clinical and histologic differences between PV and PF are accounted for by the different cell surface antigens involved. Although the final common pathway for both diseases seems to be protease release which results in acantholysis (7), the antigen involved may, in a yet undefined way, influence the location of the blister in the epidermis.

Another explanation for the different clinical and histologic expression of PV and PF is that perhaps PF and PV antibodies have the same specificity, but the reactive antigen is missing in the deeper epidermis of patients with PF (31). There is some experimental support, which is derived from indirect immunofluorescence studies on PF lesions, for this hypothesis (31). However, the present study only found one PF serum that contained antibodies that were directed against PV antigen, and that serum also contained antibodies that were directed against an antigen defined by other PF sera. Thus, patients with PF who only have antibodies against PV antigen must be unusual, if they exist at all.

The identification and further characterization of specific antigens that are defined by PV and PF autoantibodies should help define the molecular basis of these diseases, and also should ultimately advance our understanding of the similarities and differences in their pathophysiology.

\section{Acknowledgments}

We gratefully acknowledge Dr. Stephen Katz for valuable discussions, Ms. Vera Klaus-Kovtun for technical assistance, and Ms. Sybil Laird and Ms. Pat West for secretarial work.

This work was supported by a National Institutes of Health grant (1RO1 AM 31706-01) and a grant from the Uniformed Services University of the Health Sciences (R08400).

\section{References}

1. Jordon, R. E. 1979. Pemphigus. In Dermatology in General Medicine. T. B. Fitzpatrick, A. Z. Eisen, K. Wolff, I. M. Freedberg, and K. F. Austen, editors. McGraw-Hill Book Co., Inc., New York. Second ed. $310-317$.

2. Ahmed, A. R., J. Graham, R. E. Jordon, and T. T. Provost. 1980. Pemphigus: current concepts. Ann. Intern. Med. 92:396-405. 
3. Beutner, E. H., R. E. Jordon, and T. P. Chorzelski. 1968. The immunopathology of pemphigus and bullous pemphigoid. $J$. Invest. Dermatol. 51:63-80.

4. Anhalt, G. J., R. S. Labib, J. J. Voorhees, T. F. Beals, and L. A. Diaz. 1982. Induction of pemphigus in neonatal mice by passive transfer of IgG from patients with the disease. N. Engl. J. Med. 306:1189-1196.

5. Schiltz, J. R., and B. Michel. 1976. Production of epidermal acantholysis in normal human skin in vitro by the IgG fraction from pemphigus serum. J. Invest. Dermatol. 67:254-260.

6. Deng, J. S., E. H. Beutner, S. Shu, and T. P. Chorzelski. 1977. Pemphigus antibody action on skin explants. Arch. Dermatol. 113:923926.

7. Hashimoto K., K. M. Shafran, P. S. Webber, G. S. Lazarus, and K. H. Singer. 1983. Anti-cell surface pemphigus autoantibody stimulates plasminogen activator activity of human epidermal cells. A mechanism for the loss of epidermal cohesion and blister formation. J. Exp. Med. 157:259-272.

8. Bystryn, J. C., E. Abel, and C. DeFeo. 1974. Pemphigus foliaceus. Subcorneal intercellular antibodies of unique specificity. Arch. Dermatol. 110:857-861.

9. Wood, G. W., and E. H. Beutner. 1977. Blocking-immunofluorescence studies on the specificity of pemphigus autoantibodies. Clin. Immunol. Immunopathol. 7:168-175.

10. Farb, R. M., R. Dykes, and G. S. Lazarus. 1978. Anti-epidermalcell-surface pemphigus antibody detaches viable epidermal cells from culture plates by activation of proteinase. Proc. Natl. Acad. Sci. USA. 75:459-463.

11. Schiltz, J. R., B. Michel, and R. Papay. 1978. Pemphigus antibody interaction with human epidermal cells in culture. A proposed mechanism for pemphigus acantholysis. J. Clin. Invest. 62:778-788.

12. Schiltz, J. R., B. Michel, and R. Papay. 1979. Appearance of pemphigus acantholysis factor in human skin cultured with pemphigus antibody. J. Invest. Dermatol. 73:575-581.

13. Morioka, S., K. Naito, and H. Ogawa. 1981. The pathogenic role of pemphigus antibodies and proteinase in epidermal acantholysis. J. Invest. Dermatol. 76:337-341.

14. Woo, T. Y., V. A. Hogan, H. Patel, G. J. Anhalt, R. S. Labib, J. J. Voorhees, and L. A. Diaz. 1983. Specificity and inhibition of the epidermal cell detachment induced by pemphigus IgG in vitro. J. Invest. Dermatol. 81:115s-121s.

15. Stanley, J. R., M. Yaar, P. Hawley-Nelson, and S. I. Katz. 1982. Pemphigus antibodies identify a cell surface glycoprotein synthesized by human and mouse keratinocytes. J. Clin. Invest. 70:281-288.

16. Stanley, J. R., O. M. Alvarez, E. W. Bere, W. H. Eaglstein, and S. I. Katz. 1981. Detection of basement membrane zone antigens during epidermal wound healing in pigs. J. Invest. Dermatol. 77:240-243.
17. Hawley-Nelson, P., J. E. Sullivan, M. Kung, H. Hennings, and S. H. Yuspa. 1980. Optimized conditions for the growth of human epidermal cells in culture. J. Invest. Dermatol. 75:176-182.

18. Rheinwald, J. G., and H. Green. 1975. Serial cultivation of strains of human epidermal keratinocytes: the formation of keratinizing colonies from single cells. Cell. 6:331-344.

19. Fuchs, E., and H. Green. 1981. Regulation of terminal differentiation of cultured human keratinocytes by vitamin A. Cell. 25:617625.

20. Thivolet, C. H., H. H. Hintner, and J. R. Stanley. 1984. The effect of retinoic acid on the expression of pemphigus and pemphigoid antigens in cultured human keratinocytes. J. Invest. Dermatol. 82:329334.

21. Stanley, J. R., P. Hawley-Nelson, S. H. Yuspa, E. M. Shevach, and S. I. Katz. 1981. Characterization of bullous pemphigoid antigen: a unique basement membrane protein of stratified squamous epithelia. Cell. 24:897-903.

22. Laemmli, U. K. 1970. Cleavage of structural proteins during assembly of the head of bacteriophage T4. Nature (Lond.). 277:680685.

23. Bonner, W. M., and R. A. Laskey. 1974. A film detection method for tritium-labeled proteins and nucleic acids in polyacrylamide gels. Eur. J. Biochem. 46:83-88.

24. Laskey, R. A., and A. D. Mills. 1975. Quantitative film detection of ${ }^{3} \mathrm{H}$ and ${ }^{14} \mathrm{C}$ in polyacrylamide gels by fluorography. Eur. J. Biochem. $56: 335-341$.

25. Stanley, J. R., D. T. Woodley, and S. I. Katz. 1984. Identification and partial characterization of pemphigoid antigen extracted from normal human skin. J. Invest. Dermatol. 82:108-111.

26. Towbin, H., T. Staehelin, and J. Gordon. 1979. Electrophoretic transfer of proteins from polyacrylamide gels to nitrocellulose sheets: procedure and some applications. Proc. Natl. Acad. Sci. USA. 76:43504354.

27. Olmsted, J. B. 1981. Affinity purification of antibodies from diazotized paper blots of heterogeneous protein samples. J. Biol. Chem. 256:11955-11957.

28. Diaz, L. A., H. Patel, and N. J. Calvanico. 1980. Isolation of pemphigus antigen from human saliva. J. Immunol. 124:760-765.

29. Miyagawa, S., T. Hojo, J. Ishii, J. Yoshioka, and K. Sakamoto. 1977. Isolation and characterization of soluble epidermal antigens reactive with pemphigus antibodies. Acta Dermato-Venereol. 57:7-13.

30. Shu, S. Y., and E. H. Beutner. 1973. Isolation and characterization of antigens reactive with pemphigus antibodies. $J$. Invest. Dermatol. 61:270-276.

31. Bystryn, J. C., and J. Rodriguez. 1978. Absence of intercellular antigens in the deep layers of the epidermis in pemphigus foliaceus. $J$. Clin. Invest. 61:339-348. 